\title{
In-situ functionalization of aniline oligomer onto layered graphene sheet and study of its application on electrochemical detection of ascorbic acidin food samples
}

\author{
S. Devasena ${ }^{1}$, S. Meenakshi ${ }^{2}$, R. Sayeekannan ${ }^{1}$, K. Pandian ${ }^{2, *}$ \\ ${ }^{1}$ Department of Chemistry, Thiagarajar College, Madurai-625009, India \\ ${ }^{2}$ Department of Inorganic Chemistry, University of Madras, Guindy, Chennai-600025, India \\ *jeevapandian@yahoo.co.uk
}

PACS 82.47.Rs

DOI 10.17586/2220-8054-2016-7-4-774-779

\begin{abstract}
Aniline oligomers are considered as one of the electron transfer mediators for the electrochemical oxidation of ascorbic acid. The electrochemical oxidation of ascorbic acid was investigated using aniline oligomer-functionalized polymer modified electrode. In the present investigation, we demonstrated a novel methodology for the in-situ modification of aniline oligomer onto the layered graphene sheet by using diazonium salt form as precursor molecule. An enhanced electrocatalytic current was obtained for the oxidation of ascorbic acid using aniline pentamer-functionalized reduced graphene oxide (AP-rGO). Detailed studies have been carried out to study the surface modified rGO by FTIR spectroscopy. A linear relationship between peak current against the concentration of ascorbic acid was observed within the ranges from $1 \mu \mathrm{M}$ to $10 \mu \mathrm{M}$. The detection limit was measured at signal/noise $(\mathrm{S} / \mathrm{N})$ of 3 . The present method can be utilized for the electrochemical detection of ascorbic acid present in food products like fruit juices.
\end{abstract}

Keywords: graphene oxide, pentamer, voltammetric method, ascorbic acid, food samples.

Received: 3 May 2016

Revised: 25 June 2016

\section{Introduction}

Ascorbic acid is one of most important biological compounds involved in various human metabolisms. It is used to ameliorate a number of illnesses, such as the scurvy, common cold, cancer and AIDS [1]. Moreover it is present in fresh fruits, vegetables and also available in pharmaceutical products and foodstuffs as an oxidant as well as stabilizer. Thus, the development of inexpensive and easy methods for the determination of ascorbic acid is particularly important in the pharmaceutical and food industries. There are several reported methods for the detection of ascorbic acid in foodstuffs, such as chromatography [2], spectrophotometry [3], capillary electrophoresis [4] and most recently, electrochemical methods [5-7].

The amperometric determination of ascorbic acid is based on its electrochemical oxidation, which occurs at high potential at carbon or metal electrodes, however, fouling by oxidation products leads to poor reproducibility [8]. Numerous attempts to decrease the high working electrode potential and improve reproducibility have been made by modifying the electrode surface with various active mediators for the electrochemical oxidation of ascorbic acid. Attempts have been made to develop a chemically-modified electrode with various redox mediators like 7,7,8,8-tetracyanoquinodimethane, osmium 2,2-bipyridyl poly-4-vinyl pyridine chloride complex, lanthanum 2,6dichlorophenolindophenol, manganese dioxide and a cobalt (II) phthalocyanine and Prussian blue was also used to oxidize ascorbic acid $[9,10]$. The electrocatalytic oxidation of ascorbic acid on conducting polymer modified electrodes, in particular on polyaniline, has also been studied. Recently, aniline oligomer-functionalized polymers have been used for the electrochemical oxidation of ascorbic acid has been developed that has both a low applied operating potential and a low detection limit.

In the present investigation, we demonstrated the preparation of an aniline oligomer-modified graphene oxide based on chemical reductive binding of diazonium salt form of aniline oligomer in presence of graphene oxide. The electrocatalytic behavior of an aniline oligomer-functionalized graphene oxide-modified glassy carbon electrode was tested against the electrochemical oxidation of ascorbic acid in phosphate buffer solution. The greater sensitivity and low detection limits were achieved by the differential pulse voltammetry method. Thus, the present method can be considered as an efficient one for electrochemical detection of ascorbic acid in food products. 


\section{Experimental Section}

\subsection{Chemicals}

Graphite powder, Ascorbic acid and N, N-diphenylamine were received from Sigma Aldrich, USA. Ammonium hydroxide and sodium nitrite were purchased from Fisher Scientific Pvt. Ltd. India. DMF, $\mathrm{HCl}, \mathrm{H}_{2} \mathrm{SO}_{4}$ and ethanol were received from SRL Pvt. Ltd. India. Ethanol and $\mathrm{KMnO}_{4}$ were procured from Merck, India. All reagents and chemicals were used an analytical grade and without further purification.

\subsection{Preparation of pentamer}

$2.28 \mathrm{~g}$ amino capped aniline trimer and $3.04 \mathrm{~g} \mathrm{~N}, \mathrm{~N}$-diphenylamine were dissolved with $80 \mathrm{~mL}$ of DMF. $20 \mathrm{~mL}$ of water and $10 \mathrm{~mL}$ of $36 \%$ of $\mathrm{HCl}$ were then added to the above solution with vigorous stirring at $4 \mathrm{hrs}$ for room temperature. The HCl-doped aniline pentamer was obtained by filtration and then washed by a mixture of DMF/water. The product was doped in $100 \mathrm{~mL}$ of $1 \mathrm{ML}^{-1}$ ammonium hydroxide for $30 \mathrm{~m}$ to produce aniline pentamer in emeraldine state. The emeraldine aniline pentamer was reduced by phenylhydrazine and was precipitated in a $\mathrm{H}_{2} \mathrm{O}$ /ethanol mixture. The leucomeraldine aniline pentamer was collected by filtration and washed thoroughly with $\mathrm{H}_{2} \mathrm{O}$ /ethanol mixture. The product was finally dried in vaccum oven.

\subsection{Preparation of graphene oxide}

$20 \mathrm{~g}$ of graphite powder stirred with $500 \mathrm{~mL}$ of conc. $\mathrm{H}_{2} \mathrm{SO}_{4}$ in $1 \mathrm{~L}$ beaker for $30 \mathrm{~min}$. Then, $60 \mathrm{~g}$ of $\mathrm{KMnO}_{4}$ was gradually added to this solution (added $20 \mathrm{~g} / 30$ minutes). The solution was then stirred for an additional 5 to $8 \mathrm{~h}$ and then added one $1 \mathrm{~L}$ water $(900 \mathrm{~mL})$ to the above mixture. Then, the reaction was terminated by addition of $50 \mathrm{~mL} \mathrm{H} \mathrm{O}_{2}$ solution. Repeated centrifugation was done by using $5 \% \mathrm{HCl}$ aqueous solution followed by washed with deionized water until the $\mathrm{pH}$ of the solution reaches neutral. Then $160 \mathrm{~mL}$ of water was added resulting in the formation of precipitate. A uniform suspension of grapheme oxide (GO) nanoparticles was obtained after sonication [11].

\subsection{Preparation of AP-rGO}

The modification of GO was accomplished by dispersing GO $(10 \mathrm{mg})$ in DI water $(5 \mathrm{~mL})$ to which aniline pentamer $(10 \mathrm{mg})$ in $2 \mathrm{~mL}$ water was added. After complete dispersion of the reagent $0.011 \mathrm{~g}$ sodium nitrite in $1 \mathrm{ml}$ of conc. $\mathrm{HCl}$ was added drop wise. The mixture was stirred for $4 \mathrm{~h}$ and then filtered, washed successively with deionized water followed by methanol. Finally, the powdered form of the product was isolated and then dried under nitrogen atmosphere in overnight.

\subsection{Instrumentation}

FTIR spectrum was recorded in the range of 400 to $4000 \mathrm{~cm}^{-1}$ FTIR spectrum was collected against the background spectrum of $\mathrm{KBr}$. The cyclic voltammetric experiment was carried out using CHI 660A electrochemical instrument, USA and Gamry model 330, USA. A conventional three electrode system comprising of glassy carbon electrode (GCE) of $3 \mathrm{~mm}$ of geometrical surface area was purchased from BAS. Pvt. Ltd., USA. The Ag/AgCl and platinum wire were used as a reference electrode and counter electrode, respectively. The working electrode was polished using Bioanalytical system (BAS, USA) polishing kit.

\section{Results and discussion}

\subsection{Characterization of AP-rGO}

The FTIR spectrum of pentamer and AP-rGO are shown in Fig. 1. The absorption of aniline pentamer showed main bands at $1590 \mathrm{~cm}^{-1}$ and $1485 \mathrm{~cm}^{-1}$ assigned to the absorption of benzene ring and the quinoid ring. The $1273 \mathrm{~cm}^{-1}$ band was attributed to $\mathrm{C}-\mathrm{N}$ stretching in the proximity of quinoid rings as shown in Fig. 1 [12]. After modification of AP-rGO, the benzenoid and quinoid peaks are bounded in the surface of graphene oxide. So these results confirm that the pentamer modified on graphene oxide surface.

The electrochemical behavior of AP-rGO was studied and the resulting cyclic voltammetry responses are shown in Fig. 2. As seen from Fig. 2(A), the typical cyclic voltammogram of AP-rGO in presence of $0.1 \mathrm{M}$ $\mathrm{H}_{2} \mathrm{SO}_{4}$. With increase of scan rate the electrocatalytic oxidation peak current also increased gradually in the range from 5-100 mV/s. A linear relationship exists between peak current $\left(\mathrm{I}_{p}\right)$ versus scan rate $(\nu)$ and a linear regression equation of $\mathrm{y}=0.406-5.685$ with correlation coefficient $\left(\mathrm{R}^{2}\right)$ of 0.9959 as shown in Fig. 2(B), which illustrates a reversible electron transfer process with adsorption controlled one. 


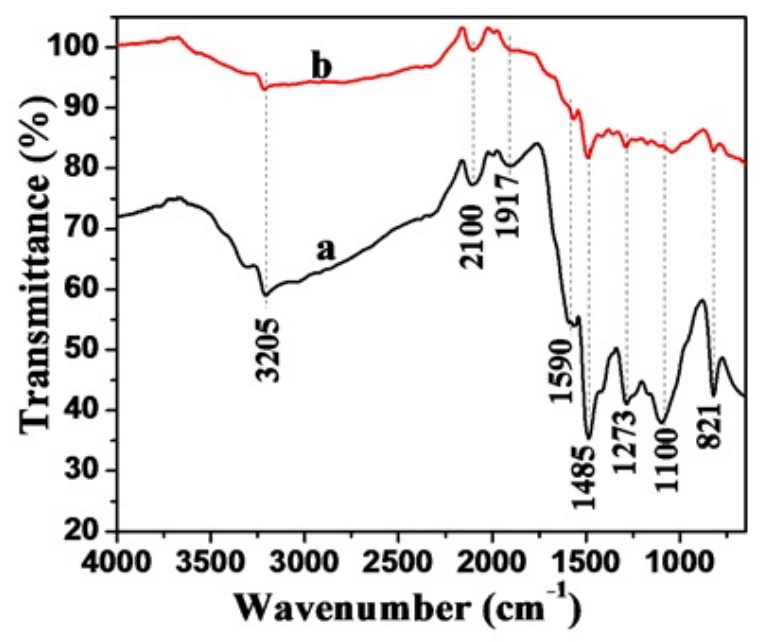

FIG. 1. FTIR spectrum of a) pentamer and b) AP-rGO
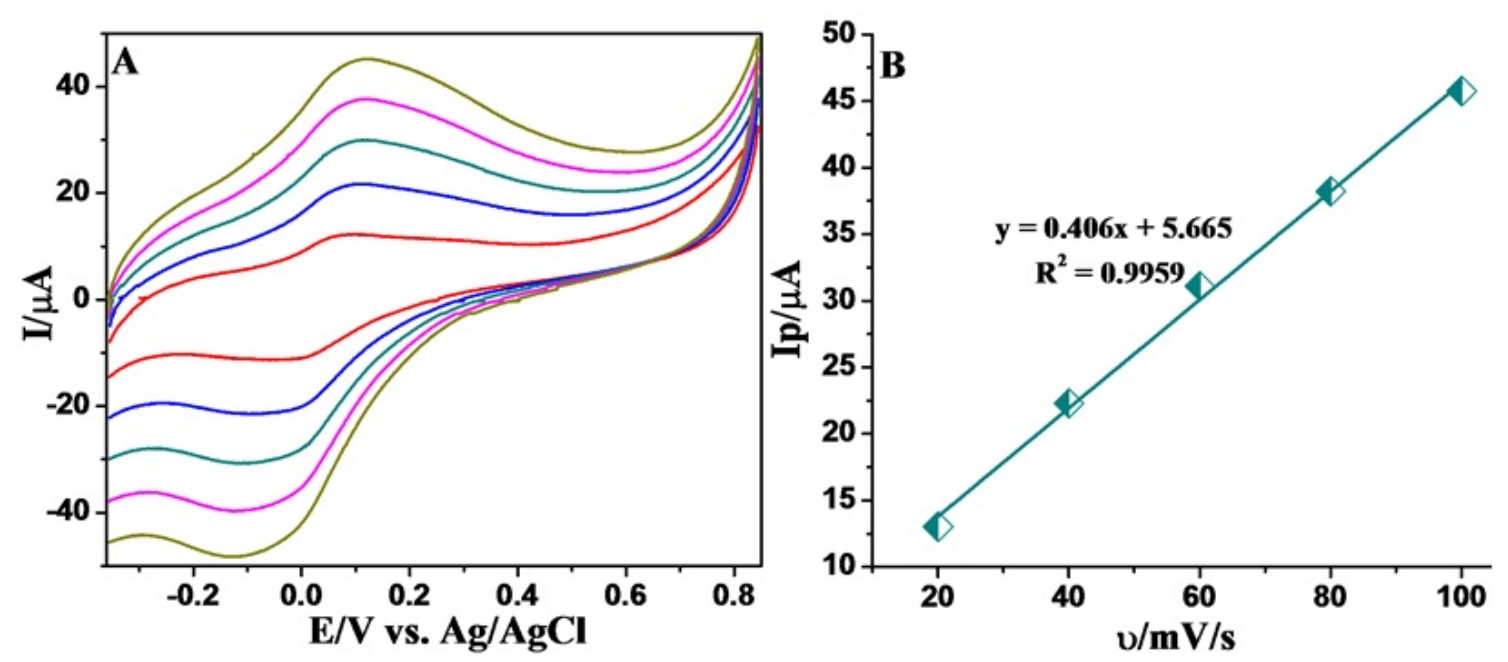

FIG. 2. A) $\mathrm{CV}$ of AP-rGO modified GCE at different scan rates $(5-100 \mathrm{mV} / \mathrm{s})$ in presence of 0.1 $\mathrm{M} \mathrm{H}_{2} \mathrm{SO}_{4}$. B) Linear plot of Ip vs. $v$

\subsection{Electrochemical oxidation of ascorbic acid}

The electrochemical behaviors of a) bare, b) bare-with ascorbic acid and c) AP-rGO modified GCE in presence of AA are shown in Fig. 3.(A) poor anodic current response was observed for the AA oxidation on bare GCE where as a well define oxidation peak was observed at $+0.05 \mathrm{~V}$ (vs. Ag/AgCl) in the case of AP-rGO modified GCE.

The electrochemical behavior of AP-rGO/GCE at different concentrations of ascorbic acid $\left(1 \times 10^{-4}-7 \times 10^{-4} \mathrm{M}\right)$ were investigated in $0.1 \mathrm{M} \mathrm{pH}$ 7.0 PBS by cyclic voltammetry (CV). As can be seen in Fig. 4(A), the AP-rGO/GCE after addition of AA to the buffer solution, an irreversible oxidation peak was observed at a scan rate of $50 \mathrm{mV} / \mathrm{s}$ with a anodic oxidation peak potential of $+0.05 \mathrm{~V}$ (vs. $\mathrm{Ag} / \mathrm{AgCl}$ ). In Fig. 4(B) shows a linear regression equation of $y=0.092 x-0.14$ with correlation coefficient is 0.9994 .

The influence of peak potential scan rate against the electrochemical oxidation of ascorbic acid was investigated using AP-rGO/GCE in presence of $1 \mathrm{mM}$ of ascorbic acid at $0.1 \mathrm{M}$ PBS ( $\mathrm{pH} 7)$ with various scan rates as shown in Fig. 4(C). From AP-rGO modified GCE, the oxidation peak current of AA was increased by increasing the scan rates $(10-120 \mathrm{mV} / \mathrm{s})$ and linearity was observed by plotting the peak current values versus the square of scan rates with correlation coefficient of 0.9920 (Fig. 4(D)), suggesting that the electrochemical oxidation of AA is diffusion controlled electron transfer process. 


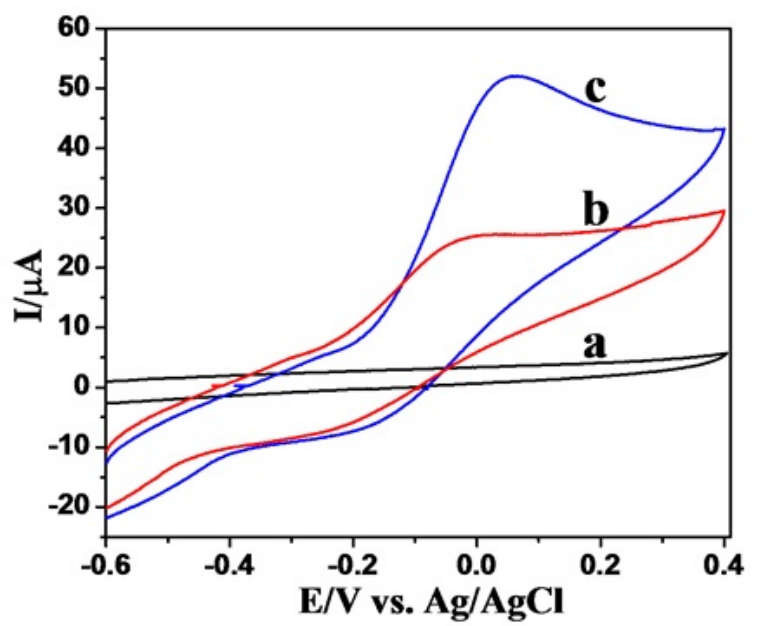

FIG. 3. Electrocatalytic behavior of a) bare, b) bare-with AA (1 mM) and c) AP-rGO/GCE with $1 \mathrm{mM}$ of AA in $0.1 \mathrm{M}$ PBS $(\mathrm{pH} 7)$ at scan rate $50 \mathrm{mV} / \mathrm{s}$
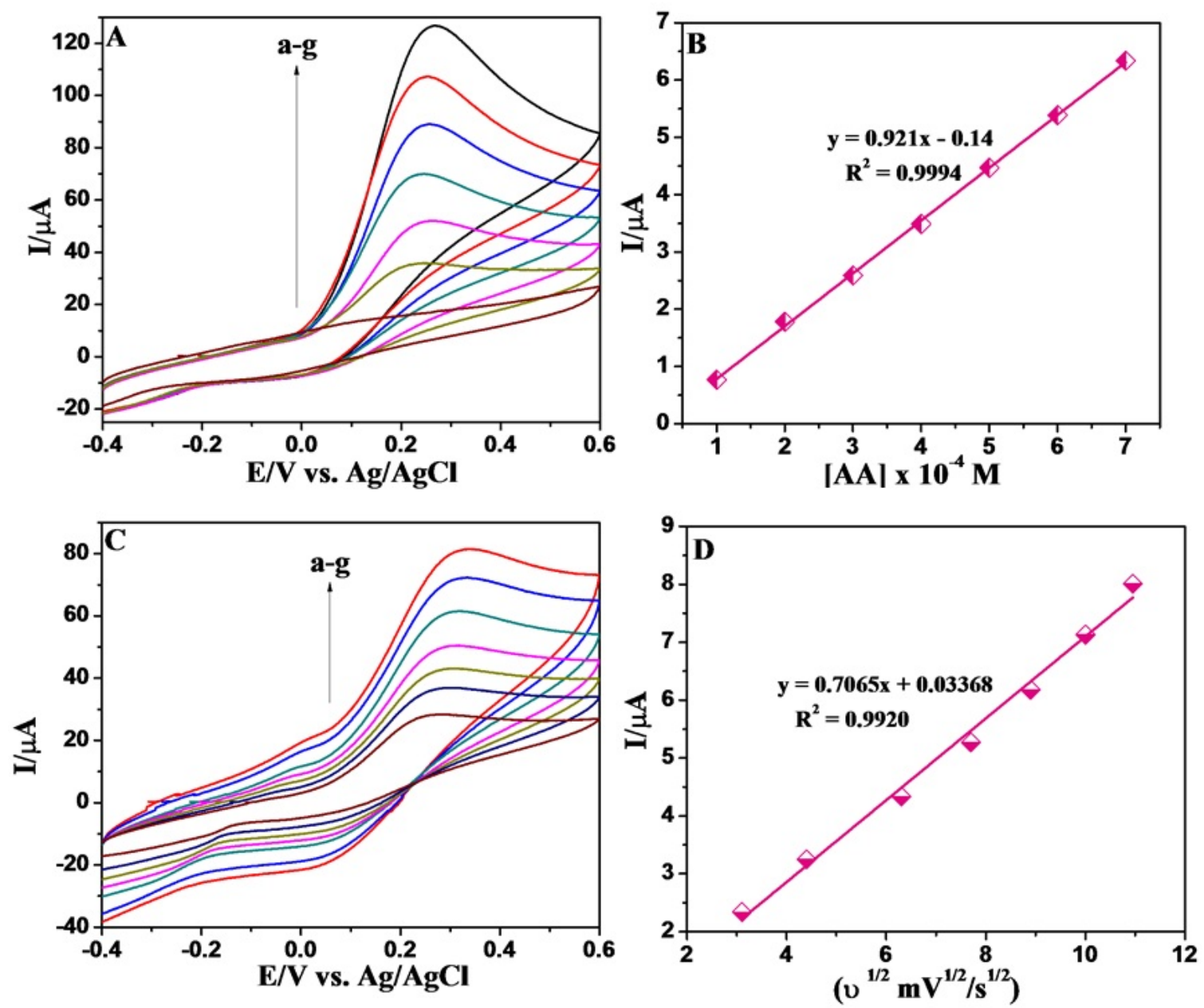

FIG. 4. A) AP-rGO/GCE in presence of $\mathrm{AA}$ in $0.1 \mathrm{M}$ PBS ( $\mathrm{pH} 7)$ at scan rate $50 \mathrm{mV} / \mathrm{s}$ and B) Linear plot of conc. vs. peak current. C) AP-rGO/GCE in presence of AA at different scan rates in $0.1 \mathrm{M}$ PBS ( $\mathrm{pH} 7)$ and D) Linear plot of Ip vs. $v^{1 / 2}$ 


\subsection{Differential pulse voltammetry method}

For the quantitative detection of ascorbic acid (AA) present in the pharmaceutical formulations and fruits samples at low concentration levels, the differential pulse voltammetry method was demonstrated using AP-rGO modified GCE under optimized experimental conditions. Fig. 5(A) shows differential pulse voltammogram for the electrocatalytic oxidation of AA using AP-rGO/GCE in PBS (pH 7) containing various concentrations of AA. The result shows the electrocatalytic peak current of AA oxidation at the AP-rGO/GCE was linearly dependent on the AA concentration and its ranges from $0.3 \mu \mathrm{M}$ to $3 \mu \mathrm{M}$. As seen from Fig. 5(B), the calibration plot shows electrocatalytic peak current $\left(\mathrm{I}_{p}\right)$ versus AA concentrations with linear regression equation of $y=0.494 x-0.212$ with correlation coefficient of 0.9923 and the detection limit ( $3 \sigma /$ slope, $\sigma$ is a standard deviation) is found to be $20 \mathrm{nM}$. These results strongly suggest that the oxidation of AA can be good selective and sensitive at aniline AP -rGO/GCE.
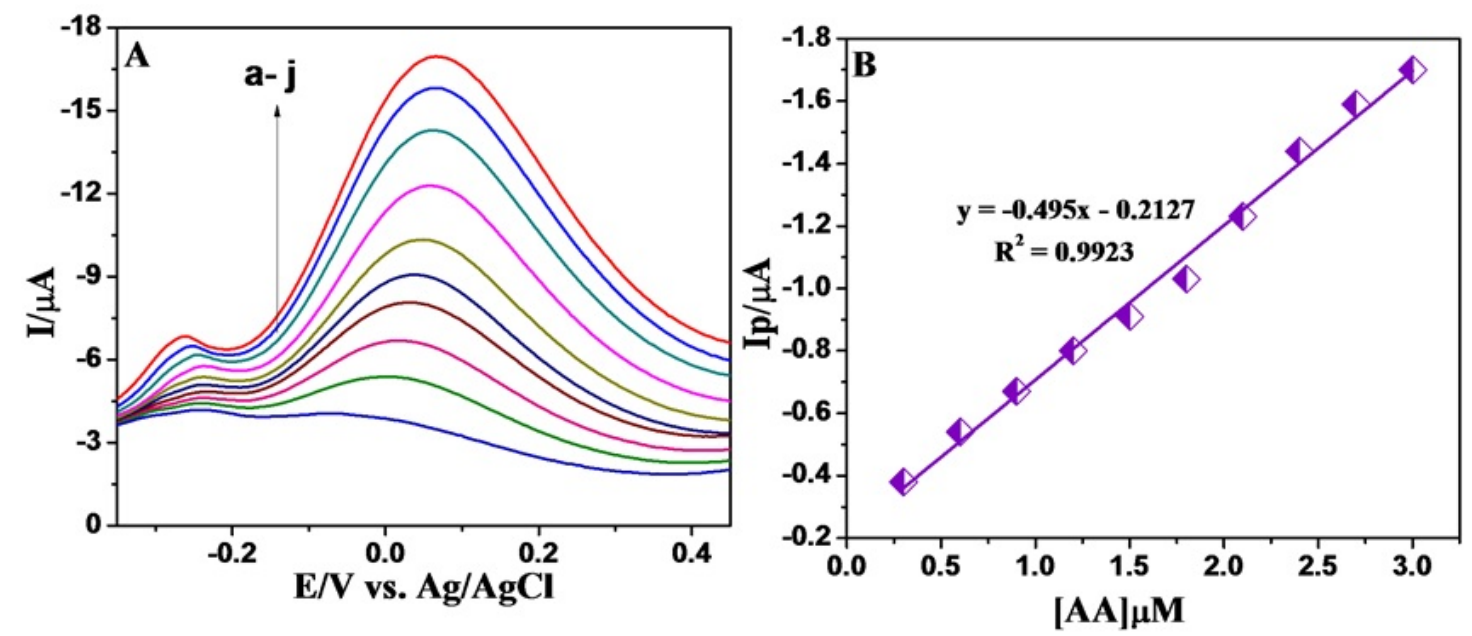

FIG. 5. A) DPV of $\mathrm{AP}-\mathrm{rGO} / \mathrm{GCE}$ in presence of $\mathrm{AA}$ at various concentrations $(0.3-3.0 \mu \mathrm{M})$ in $0.1 \mathrm{M}$ PBS ( $\mathrm{pH} 7)$. B) Linear plot of concentration vs. peak current

\subsection{Food sample analysis}

In order to estimate the amount of AA present, fruit juice food samples were analyzed. Based on the repeated differential pulse voltammetric responses $(n=5)$ of the diluted analyte, samples were spiked with specified concentration of AA, measurements were made for determination of AA concentration in food samples. The results are listed in Table 1. The AP-rGO/GCE possessed reasonable selectivity and produced satisfactory recovery result with an average recovery of $100.3 \%$ and the RSD was less than $5.3 \%$. The measured data of AA concentration was in good agreement with the results found in the titration measurements, suggesting the good accuracy and reliability.

TABLE 1. Determination of AA in fruit samples

\begin{tabular}{|c|c|c|c|}
\hline Samples & Added $\left(\mathbf{1 0}^{-6} \mathbf{g} / \mathbf{m l}^{-1}\right)$ & Found $\left(\mathbf{1 0}^{-6} \mathbf{g} / \mathbf{m l}^{-1}\right)$ & Recovery $\mathbf{( \% )}$ \\
\hline Apple & 0.50 & 1.024 & 99.3 \\
\hline Pineapple & 0.70 & 1.208 & 100.6 \\
\hline Orange & 1.50 & 2.133 & 101 \\
\hline
\end{tabular}

\section{Conclusion}

A single step method was adapted to modified graphene sheet with aniline pentamer as electron transfer mediator. The surface modification of graphene was confirmed from FTIR and cyclic voltammetry studies. A simple differential pulse voltammetry method was developed to determine low concentration ranges of ascorbic acid using aniline pentamer grafted graphene sheet. Enhanced detection limits was achieved using this method. The present method can be utilized for the electrochemical detection of ascorbic acid in pharmaceutical and fruit samples. 


\section{Acknowledgement}

The authors (Dr. K. P and S. M.) are grateful to DST purse programme to upgrade the Gamry 330 electrochemical system (DPV software).

\section{References}

[1] DeNobili M.D., Perez C.D., Navarro D.A., Stortz C.A., Rojas A.M. Hydrolytic stability of L-(+)-ascorbic acid in low methoxyl pectin films with potential antioxidant activity at food interfaces. Food Bioprocess Technol., 2011, 6, P. 186-197.

[2] Jens L. Determination of ascorbic acid and dehydroascorbic acid in biological samples by high performance liquid chromatography using subtraction methods: Reliable reduction with tris (2-carboxyethyl) phosphine hydrochloride. Anal. Biochem., 2000, 282, P. 89-93.

[3] Yu Y.H., Donat P.H. UV-B-induced formation of reactive oxygen species and oxidative damage of the cyanobacterium Anabaena sp.: protective effects of ascorbic acid and N-acetyl-L-cysteine. J. Photochem. Photobio. B: Biology, 2002, 66, P. 115-124.

[4] $\mathrm{Wu}$ T., Guan Y., Ye J. Determination of flavonoids and ascorbic acid in grapefruit peel and juice by capillary electrophoresis with electrochemical detection. Food Chem., 2007, 100, P. 1573-1579.

[5] Ping J., Wang Y., Wu J., Ying Y., Ji F. Determination of ascorbic acid levels in food samples by using an ionic liquid carbon nanotube composite electrode. Food Chem., 2012, 135, P. 362-367.

[6] Francis F.J. Wiley encyclopedia of food science and technology, 2-nd ed., Vol 4. Wiley, New York, 1999.

[7] Habibi B., Pournaghi-Azar M.H. Simultaneous determination of ascorbic acid, dopamine and uric acid by use of a MWCNT modified carbon-ceramic electrode and differential pulse voltammetry. Electrochimica Acta, 2012, 55, P. 5492-5498.

[8] Roy P.R., Saha M.S., Okajima T., Ohsaka T. Electrooxidation and amperometric detection of ascorbic acid at GC electrode modified by electropolymerization of N, N-Dimethylaniline. Electroanalysis, 2004, 16, P. 289-297.

[9] Florou A.B., Prodromidis M.I., Stella M.T.K., Miltiades I. Fabrication and voltammetric study of lanthanum 2,6-dichlorophenolindophenol chemically modified screen printed eletrodes: Application for the determination of ascorbic acid. Anal. Chimica Acta, 2000, 423, P. 107114

[10] Zuo X., Zhang H., Li N. An electrochemical biosensor for determination of ascorbic acid by cobalt (II) phthalocyanine- multiwalled carbon nanotubes modified glassy carbon electrode. Sensors Actuat., 2012, 161, P. 1074-1079.

[11] Hummers W.S., Offemn R.E. Preparation of graphitic oxide. J. Am. Chem. Soc., 1958, 80, P. 1339.

[12] Huang L., Hu J., Lang L., Wang X., Zhang P., Jing X., Wang X., Chen X., Lelkes P.I., MacDiarmid A.G., Wei Y. Synthesis and characterization of electroactive and biodegradable ABA block copolymer of polylactide and aniline pentamer. Biomaterials, 2007, 28, P. $1741-1751$ 